\author{
Mary Ann Liebert, Inc. Fublishers
}

\title{
A Population-Based Study of Pre-Existing Health Conditions in Traumatic Brain Injury
}

\author{
Kristine C. Dell, ${ }^{1,2}$ Emily C. Grossner, ${ }^{1,2}$ Jason Staph, ${ }^{1}$ Philip Schatz, ${ }^{3}$ and Frank G. Hillary ${ }^{1,2,4^{*}}$
}

\begin{abstract}
Health factors impacting both the occurrence of, and recovery from traumatic brain injury (TBI) vary in complexity, and present genuine challenges to researchers and healthcare professionals seeking to characterize injury consequences and determine prognosis. However, attempts to clarify causal links between injury characteristics and clinical outcomes (including mortality) often compel researchers to exclude pre-existing health conditions (PECs) in their samples, including psychiatric history, medication usage, and other comorbid conditions. In this pre-registered population-based study (total starting $n=939,123$ patients), we examined trends in PEC incidence over 22 years in the state of Pennsylvania (1997-2019) in individuals sustaining TBI $(n=169,452)$ and individuals with orthopedic injury $(n=87,637)$. The goal was to determine how PECs interact with age and injury severity to influence short-term outcomes. A further goal was to determine whether number of PECs, or specific PEC clusters contributed to worse outcomes within the TBI cohort, compared with orthopedic injury alone. Primary findings indicate that PECs significantly influenced mortality within the TBI cohort; patients having four or more PECs were associated with approximately a two times greater likelihood of dying in acute care (odds ratio [OR] 1.9). Additionally, cluster analyses revealed four distinct PEC clusters that are age and TBI severity dependent. Overall, the likelihood of zero PECs hovers at $\sim 25 \%$, which is critical to consider in TBI outcomes work and could potentially contribute to the challenges facing intervention science with regard to reproducibility of findings.
\end{abstract}

Keywords: cluster analysis; pre-existing conditions; traumatic brain injury

\section{Introduction}

Despite the dense literatures examining the role of demographic and injury related/severity factors that influence early recovery from traumatic brain injury (TBI), ${ }^{1-8}$ pre-existing health conditions (PECs) are relatively under-studied outcomes. Health conditions co-occurring with injury increase as a function of advancing age, and contribute to variability in both post-injury trajectories as well as heterogeneous factors involved in recovery. ${ }^{9-14}$ Emerging research has demonstrated that comorbidities can complicate functional recovery and symptom resolution. However, these studies frequently focus on select (notably psychiatric) PECs, or examine the contribution of TBI to exacerbated systemic disorders or illness rather than the opposing directionality. ${ }^{15,16}$ Similarly, incongruity in the terminology utilized to discuss PECs, combined with diversity in identification and classification methods of these conditions (self-report, extraction from medical records, semi-structured interview), can also complicate our ability to reconcile findings from existing studies. For our purposes, the term PEC is used to signify conditions or disorders present, self-reported, or otherwise diagnosed before the time of injury. This is

\footnotetext{
${ }^{1}$ Department of Psychology, ${ }^{2}$ Social and Life and Engineering Sciences Imaging Center, The Pennsylvania State University, University Park, Pennsylvania, USA. ${ }^{3}$ Department of Psychology, Saint Joseph's University, Philadelphia, Pennsylvania, USA.

${ }^{4}$ Department of Neurology, Hershey Medical Center, Hershey, Pennsylvania, USA.

*Address correspondence to: Frank G. Hillary, PhD, Department of Psychology, The Pennsylvania State University, 313 Bruce V. Moore Building, University Park, PA 16802, USA; E-mail: fhillary@psu.edu
}

(C) Kristine C. Dell et al., 2021; Published by Mary Ann Liebert, Inc. This Open Access article is distributed under the terms of the Creative Commons License (CC-BY) (http://creativecommons.org/licenses/by/4.0), which permits unrestricted use, distribution, and reproduction in any medium, provided the original work is properly credited. 
in contrast to "nosocomial conditions," which are healthcare-associated conditions originating during or as a result of a hospital stay, ${ }^{17-19}$ and "comorbid" conditions such as substance abuse and psychiatric response to injury that are the focus months to years after the injury occurred. ${ }^{13,14,20-24}$

The Centers for Disease Control and Prevention's (CDC's) National Center for Chronic Disease Prevention and Health Promotion (NCCDPHP) estimates that 6 out of every 10 adults (18 years or older) in the United States have at least one chronic disease, and 4 out of 10 adults have at least two chronic conditions. ${ }^{25}$ Over the last 10 years, cardiac conditions, psychiatric conditions, diabetes, respiratory disorders, and musculoskeletal conditions have remained stable as the most frequent chronic conditions. ${ }^{26}$ Further, the NCCDPHP denotes heart disease, dementias, diabetes, arthritis, and cancer as the leading contributors of disability, death, and healthcare costs in the United States, with age conferring increased risk of these disease conditions. $^{25}$

Understanding the influence of the increased disease burden introduced by the development and progression of these PECs is critical across all severities of TBI. ${ }^{15,16,27}$ In addition, the impact of PECs does not appear limited to the acute period following TBI. Patients with moderate-to-severe TBI (msTBI) with a greater total disease burden reported reduced levels of functioning and life satisfaction up to 10 years following injury. ${ }^{28}$ Together, these studies add to the growing literature examining the impacts of both psychiatric ${ }^{1,10,13,14,20-23,29}$ and other medical conditions $^{24,30-34}$ on outcomes following TBI. Such multimorbidity adds complexity to the care required for these TBI patients in comparison to individuals with singular or no reported PECs and provides evidence for a systemic disease burden on the individual. Similarly, clinical care focused on a single diagnosis may not accurately depict the patient's comprehensive clinical picture or rehabilitative needs. ${ }^{15,24,35-37}$

In patients older than age 50 years with msTBI, hypertension emerged as a primary PEC and along with related medical complications, predicted hospitalization at 1 year post-injury. ${ }^{27}$ In elderly patients with TBI, not only are PECs associated with worse outcomes, but morbidity is more highly linked to PEC complications than complications from the injury. ${ }^{27,38}$ In a cohort of elderly patients with TBI following treatment in a neurosurgical department, patients with comorbid cardiac, pulmonary, or renal conditions and malignancy were almost three times more likely to die or enter a vegetative state post-injury. ${ }^{39}$ Related work has shown that patients age 50 years or older are significantly more likely to have diabetes, elevated cholesterol, osteoarthritis, and hypertension in comparison with individuals under the age of 50 who sustain TBI. ${ }^{40}$

Although it may be difficult to directly link the onset of these conditions post-injury as a result of TBI or aging, what does emerge is evidence for reciprocal impacts of central and peripheral nervous system functioning, ${ }^{19,41-43}$ and how this interplay may confer risk of pathological aging in the form of subsequent injury, disease progression, or exacerbated recovery. ${ }^{30,44-46}$ Studying TBI without understanding or incorporating PECs not only has broad implications for the reproducibility of findings, ${ }^{47}$ but also risks undermining the detection of important pre-injury and recovery phenotypes. $^{28,48}$ Similarly, it may also play a role in the near complete failure in advancing phase 2 clinical trials for brain injury interventions. ${ }^{41,49,50}$

\section{Study goal}

Given the importance of understanding how the presence of PECs can contribute to the heterogeneity of TBI recovery trajectories, we conducted a population-based study to identify the base rates of PECs in TBI, to examine the influence of PECs on TBI outcome, and to confirm the previously identified prevalence of PECs in individuals with TBI. Although we examined all cases of TBI during those years, we focused on moderate and severe TBI in analyses to determine the interaction between PEC and injury severity in predicting mortality and functional outcomes. We also sought to determine if PECs interact specifically with moderate and severe TBI compared with orthopedic injury to confer specific risk of prolonged hospital stay and diminished functional status at discharge.

\section{Hypotheses}

Pre-registered hypotheses

1. Mortality rate and days spent in the hospital will be positively correlated with the quantity of PECs.

2. Patients with TBI with a greater number of PECs will perform worse on measures of independence, compared with patients with TBI with fewer PECs.

3. PEC subtypes will be associated with poor outcomes in elderly patients with TBI. 
Exploratory questions (all developed

before data analysis)

1. What can the results of K-modes clustering demonstrate regarding specific characteristics of patients with TBI presenting with a higher number of PECs?

2. Does TBI (as opposed to trauma-only) select for certain PECs when examining all patients?

3. Does the frequency of PEC profiles change over the lifespan?

\section{Methods}

Beginning in October 1986, the Pennsylvania Trauma Systems Foundation (PTSF) initiated a trauma registry (Pennsylvania Trauma Outcomes Study, or PTOS) to receive data from participating hospitals, in an effort to evaluate patient outcomes through research and education across comparable centers state-wide. At the authors' request, the PTSF provided de-identified data collected from 1986 to June 2019 comprising two approximately equal-sized patient cohorts (total $n=939,123$ cases): trauma patients entering emergency departments (EDs) with a documented TBI $(n=476,006)$, and a second group of patients without a documented TBI at admission (orthopedic control group, $n=463,117)$. The TBI and orthopedic injury cases were selected according to codes specific to TBI (International Classification of Diseases, 9th Revision [ICD9] code prefixes: 800-806, 850-854, 950, 951, 952; ICD10 codes: S02, S04, S06, S07, S12, S14.0S14.3, S22.0-S22.089, S32.0-S32.3, S34.0-S34.2, S24.0-S24.2). When the PTSF provided the data, they included the binary variable "TBI" denoting patients with a diagnosis code from the requested code range, whereas patients with orthopedic injury were those without any of the listed codes. Due to the size of the raw dataset (2.1 GB, 1.2 billion cells of data), data cleaning and analysis required a multistep process implemented through several software applications.

For the framework outlined below, version control and data provenance were performed through git $^{51}$ and git annex. ${ }^{52}$ Data were received from the PTSF in two CSV formatted files, one for each requested cohort (TBI and orthopedic injury). Simple CSV validity checks were performed on the data with csv-kit, ${ }^{53}$ then the two cohort files were converted to TSV to increase speed and efficiency of use, and then were combined. Initial evaluation of the data was performed via a
Docker $^{54}$ based Elasticsearch ${ }^{55}$ and Kibana ${ }^{56}$ configuration, using $\operatorname{Logstash}^{57}$ to consume the raw data, and to index the resulting records to Elasticsearch. ${ }^{55}$ Kibana $^{56}$ was used as an explorational tool for the data and data cleaning, and normalization was then implemented using a combination of Python $^{58}$ and $\mathrm{R} .{ }^{59}$ All codes used to alter the data were tracked in git ${ }^{51}$ with original source data all subsequent revised versions tracked through git annex. ${ }^{52}$ Docker $^{54}$ compose configuration files were created to automate the use of the Elasticsearch ${ }^{55}$ and Kibana ${ }^{56}$ tools to provide consistency in the software environment across all computing resources.

To facilitate further processing and reporting on the data, sub-datasets were created through selection of limited numbers of columns, selection of records based on column values (post-cleaning), and normalization of the data within columns (converting " $<\mathrm{UNK}>$ " and " $<\mathrm{n} / \mathrm{a}>$ " to either null values, or the appropriate integer value for the column in which they were discovered). For data fields of interest, all unique values present in the data were collated, and compared with the "2017 Pennsylvania Trauma Systems Foundation Operational Manual for the Database Collection System" 60 for compliance with data entry guidelines. Data cleaning steps were applied where the unique values differed from the operational manual's allowed values, in the following ways:

1. "<n/a>," "<UNK $>$," and "-," were set to “-1," -2 ," and " -3 ," respectively, in integer fields.

2. " $<\mathrm{UNK}>$ " and " $<\mathrm{n} / \mathrm{a}>$ " were replaced with an empty string in text fields.

3. Date and Time fields were converted from several conventions to conform to ISO 8601 format. If the date or time was " $<\mathrm{UNK}>$ " or " $<\mathrm{n} / \mathrm{a}>$," it was converted to an empty string.

After list-wise deletion of cases with missing data (Fig. 1), 257,089 cases were available for use in the present analyses dating from 1997 until 2019.

\section{K-modes clustering analysis to establish} PEC profiles

Given that the PTOS permits coding of up to 10 distinct PEC entries, we elected to include all patients $(n=257,089)$, regardless of the presence of TBI or injury severity together, to implement a data-driven approach (K-modes clustering algorithm using the $k_{l a R^{61}}$ package in $\left.\mathrm{R}\right)^{59}$ to aggregate clusters of PEC profiles. Only a binary matrix representing the presence or 


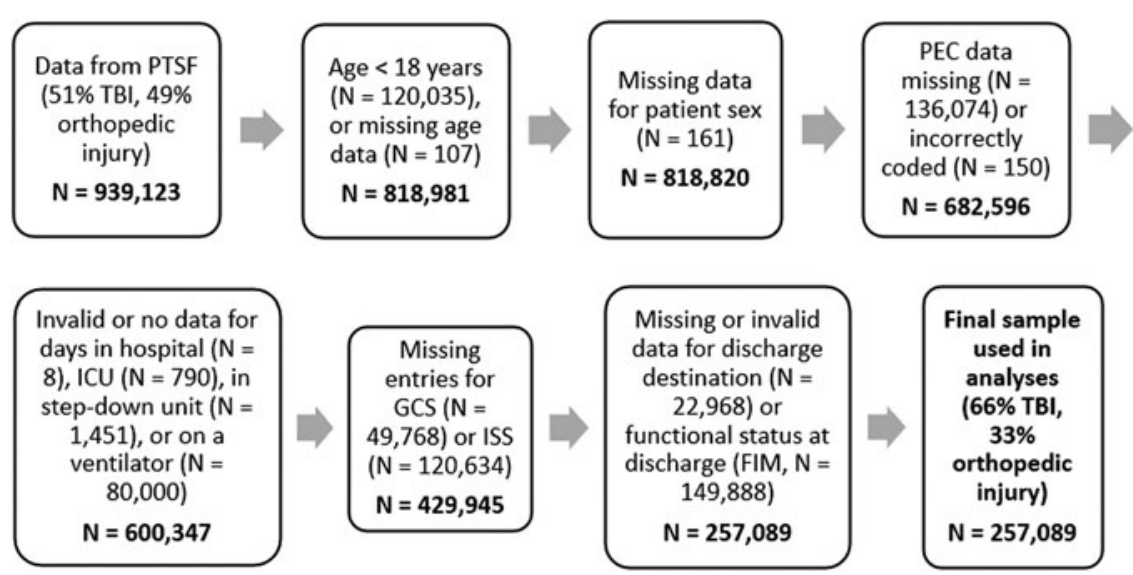

FIG. 1. List-wise deletion of cases with missing data during data cleaning. Each box identifies reasons cases were moved to create the final sample used for analysis. FIM, functional independence measure; GCS, Glasgow Coma Scale; ICU, intensive care unit; ISS, injury severity score; PEC, pre-existing condition; PTSF, Pennsylvania Trauma Systems Foundation; TBI, traumatic brain injury.

absence of each PEC for each patient was included in the K-modes analysis; demographic or injury variables were not included. We then sought to validate the optimal number of clusters in the manner consistent with one of the traditional validation methods of examining elbow plots of the within-cluster sum of squares (wss) implemented in $\mathrm{K}$-means clustering, ${ }^{62}$ given $\mathrm{K}$-modes has been presented as an extended application of the original K-means algorithm. ${ }^{63,64}$ Within the $\mathrm{K}$-modes function, we set an initial seed randomly at the value of (12345), estimated an initial number of clusters (K) equal to 4 , and requested a maximum of 10 iterations. To confirm the optimal number of clusters, we repeated these steps, with the exception of changing the value of $\mathrm{K}$ to 3,5 , and then 6 , and subsequently plotted the wss values returned by the K-modes function for each of our runs. The resulting plots are those presented in Figure 2.

A comprehensive list of the 19 PEC categories, which the PTOS defines as "pre-existing comorbid factors present before patient arrival at the Emergency Department (ED)/hospital," are provided in Supplementary Table S1 and the PTOS data manual. ${ }^{60}$ Of note, several of the PECs align with and use the same definition as the National Trauma Data Bank (NTDB) data dictionary. ${ }^{60}$ Further, during the process of data cleaning, the PEC category of Pregnancy was dropped from the dataset to remove bias in PEC count for female trauma patients represented in the database, leaving a total of 18 PEC categories for the current analyses across 43 different participating trauma centers throughout the state.

After filtering out mild TBI (mTBI) cases (Glasgow Coma Scale [GCS] score $12-15 ; n=150,721)$ and orthopedic injury cases $(n=87,633)$, we conducted logistic regression with total count of PECs as the independent variable for hypotheses examining the impact of PECs on mortality in patients with msTBI ( $n=18,729)$, using the $g l m$ function in $\mathrm{R}^{59}$ To ensure comparable cell sizes for the logistic regression, we collapsed PEC quantities 6 through 10 into one group with a PEC count value of 6 or greater $(\geq 6)$. For the remaining hypotheses regarding functional independence (FIM) and other hospital outcomes variables (days spent in the hospital, days in the intensive care unit [ICU]), we conducted linear regression on each of the three dependent variables with either PEC count or K-modes cluster assignment as the independent variables.

\section{Results}

Demographics for all subjects are presented in Table 1. Due to the high degree of missingness for the Ethnicity variable specifically $(30-90 \%)$, it was not feasible to make reliable calculations for participants of Hispanic/Latino descent. 


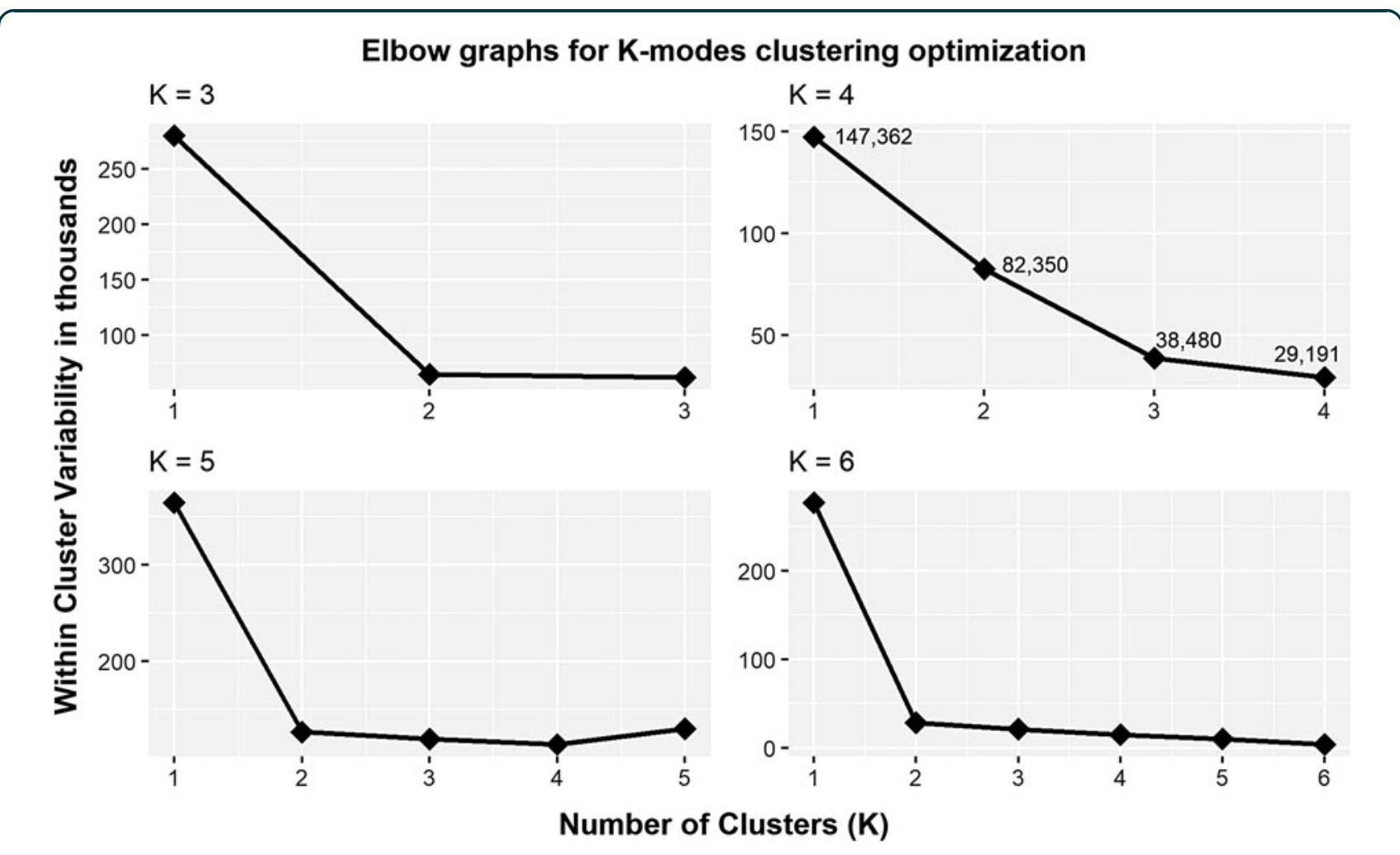

FIG. 2. Elbow plots for K-modes clustering analysis, plotting within cluster variability values across numbers of clusters set to $K=3,4,5$, or 6 . Visual inspection of these four plots confirmed $K=4$ as the optimal number of clusters.

Hypothesis 1: PECs will influence mortality and hospital outcomes

We analyzed mortality within a cohort of adult patients with msTBI ( $n=50,574$; discharged alive $=73 \%)$. Logistic regression revealed a significant overall effect of PEC quantity in predicting mortality $\left(\chi^{2}[6, n=50,574]=\right.$
427.3, $p<0.001)$. Individual Wald's tests for each level of PEC confirmed each additional PEC significantly increased the odds of patients with msTBI being discharged dead in comparison with patients with msTBI admitted with zero PECs. When comparing patients with TBI without any PECs (odds ratio,

Table 1A. Demographics of Patients with and without Recorded PEC Data Prior to Data Cleaning

\begin{tabular}{|c|c|c|c|c|c|c|}
\hline Data & Race $^{a}$ & $\%$ of $n$ & $\bar{M}$ age in years (SD) & Male & Discharged alive & Orthopedic \\
\hline $\begin{array}{l}\text { Adult patients with PEC data available at project start: } \\
\qquad N=796,756 \\
52 \% \text { TBI } \\
\text { Median GCS score }=15 \\
45 \% \text { have zero PECs }\end{array}$ & $\begin{array}{l}\text { Caucasian } \\
\text { African-American } \\
\text { Asian } \\
\text { Other } \\
\text { Unknown }\end{array}$ & $\begin{array}{l}78 \% \\
15 \% \\
0.8 \% \\
2.3 \% \\
3.2 \%\end{array}$ & $\begin{array}{l}55.3(22.8) \\
40.7(18.5) \\
49.7(21.2) \\
42.2(19.2) \\
44.1(20.2)\end{array}$ & $\begin{array}{l}58 \% \\
73 \% \\
58 \% \\
72 \% \\
72 \%\end{array}$ & $\begin{array}{l}95 \% \\
92 \% \\
94 \% \\
94 \% \\
94 \%\end{array}$ & $\begin{array}{l}46 \% \\
59 \% \\
44 \% \\
49 \% \\
52 \%\end{array}$ \\
\hline $\begin{array}{l}\text { Adult patients w/out PEC data available at project start }{ }^{\text {b: }} \\
\quad N=39,771 \\
48 \% \text { TBI } \\
\text { Median GCS }=3\end{array}$ & $\begin{array}{l}\text { No data } \\
\text { Caucasian } \\
\text { African-American } \\
\text { Asian } \\
\text { Other }\end{array}$ & $\begin{array}{l}0.2 \% \\
67 \% \\
25 \% \\
1.3 \% \\
3.2 \%\end{array}$ & $\begin{array}{l}45.6(20.9) \\
45.4(21.1) \\
34.9(16.5) \\
40.6(16.8) \\
36.2(16.3)\end{array}$ & $\begin{array}{l}65 \% \\
67 \% \\
84 \% \\
74 \% \\
83 \%\end{array}$ & $\begin{array}{l}94 \% \\
77 \% \\
55 \% \\
68 \% \\
73 \%\end{array}$ & $\begin{array}{l}49 \% \\
41 \% \\
57 \% \\
42 \% \\
42 \%\end{array}$ \\
\hline & No data & $3.3 \%$ & $40.0(21.4)$ & $85 \%$ & $47 \%$ & $47 \%$ \\
\hline
\end{tabular}

${ }^{a}$ Race and ethnicity data were not available for all patients. Due to the high degree of missingness for the Ethnicity variable, percentages of patients of Latino/Hispanic origin could not be reliably calculated.

${ }^{b}$ Patients without PEC data excluded from further analysis.

GCS, Glasgow Coma Scale; PEC, pre-exisiting health condition; SD, standard deviation; TBI, traumatic brain injury. 
Table 1B. Sub-Sample Demographics following Data Cleaning

\begin{tabular}{llrcccl}
\hline Final data samples & \multicolumn{1}{c}{ Race $^{a}$} & \% of $\mathbf{n}$ & $\bar{M}$ age in years (SD) & Male & Discharged alive & Most frequent discharge destination \\
\hline Mild TBI: & Caucasian & $84 \%$ & $56.2(22.8)$ & $59 \%$ & $100 \%$ & Home \\
$N=150,721$ & African-American & $9 \%$ & $43.3(18.6)$ & $73 \%$ & $100 \%$ & Home \\
$27 \%$ have zero PECs & Asian & $0.9 \%$ & $51.7(21.4)$ & $57 \%$ & $100 \%$ & Home \\
& Other & $2.1 \%$ & $43.7(19.7)$ & $70 \%$ & $100 \%$ & Home \\
Moderate TBI: & No data & $4 \%$ & $45.2(20.2)$ & $71 \%$ & $100 \%$ & Home \\
$N=5839$ & Caucasian & $76 \%$ & $54.0(23.5)$ & $62 \%$ & $100 \%$ & Home, rehab facility \\
$26 \%$ have zero PECs & African-American & $14 \%$ & $43.1(18.1)$ & $78 \%$ & $100 \%$ & Home, rehab facility \\
& Asian & $1.6 \%$ & $50.6(23.2)$ & $62 \%$ & $100 \%$ & Home, rehab facility \\
Severe TBI: & Other & $3 \%$ & $43.4(20.1)$ & $79 \%$ & $100 \%$ & Home, rehab facility \\
$N=12,890$ & No data & $5.4 \%$ & $43.4(20.1)$ & $73 \%$ & $100 \%$ & Home, rehab facility \\
$38 \%$ have zero PECs & Caucasian & $82 \%$ & $41.2(18.8)$ & $74 \%$ & $100 \%$ & Rehab facility, LTC \\
& African-American & $10 \%$ & $39.8(16.6)$ & $83 \%$ & $100 \%$ & Home, rehab facility \\
& Asian & $0.9 \%$ & $42.6(17.5)$ & $60 \%$ & $100 \%$ & Rehab facility, home \\
Orthopedic Injury: & Other & $2.8 \%$ & $37.0(16.4)$ & $80 \%$ & $100 \%$ & Home, rehab facility \\
$N=87,633$ & No data & $4.8 \%$ & $38.0(16.9)$ & $80 \%$ & $100 \%$ & Home, rehab facility \\
$30 \%$ have zero PECs & Caucasian & $77 \%$ & $54.2(21.8)$ & $60 \%$ & $100 \%$ & Home \\
& African-American & $16 \%$ & $37.4(16.6)$ & $77 \%$ & $100 \%$ & Home \\
& Asian & $0.8 \%$ & $46.9(20.1)$ & $61 \%$ & $100 \%$ & Home \\
& Other & $2.2 \%$ & $40.2(17.3)$ & $74 \%$ & $100 \%$ & Home \\
& No data & $4 \%$ & $40.3(18.1)$ & $75 \%$ & $100 \%$ & Home \\
\hline
\end{tabular}

${ }^{\text {a}}$ Race and ethnicity data were not available for all patients. Due to the high degree of missingness for the Ethnicity variable, percentages of patients of Latino/Hispanic origin could not be reliably calculated.

${ }^{b}$ Discharge destinations were equal in frequency.

LTC, long term care facility; PEC, pre-existing health condition; SD, standard deviation; TBI, traumatic brain injury.

$[\mathrm{OR}]=0.28)$, the ORs for TBI cases with one or more diagnosed PEC were (from 1 to 6 PECs): 1.3, 1.5, 1.6, $1.9,1.7$, and 1.7, respectively. For example, individuals with 4 PECs were nearly twice (1.9 times) more likely to die compared with those with no PECs. Separate Wald's tests also confirmed that the differences in the coefficients for each additional level of PEC (0 versus 1,1 versus 2,2 versus 3 , etc.) were all statistically significant, with the exception of the difference in coefficients for PEC quantities 5 and $6\left(\chi^{2}[1, n=2328]=0.032\right.$, $p=0.86)$.

Hypothesis 2: Number of PECs will influence outcomes in patients with TBI

The percentages of patients across injury sub-samples reported for each PEC category are presented in Figure 3. The PEC counts of patients with msTBI significantly predicted FIM scores $(F[1,18729]=206.1$, $\left.p<0.001, \eta^{2}=0.013\right)$. For each additional PEC, FIM scores were predicted to decrease by half a point $(b=-0.5, t \quad[18729]=-14.4, p<0.001)$. Similarly, PEC counts of patients with msTBI significantly predicted total days spent in the ICU, $(F[1,18729]=9.3$, $\left.p=0.002, \eta^{2}=0.001\right)$. More specifically, each additional PEC is expected to decrease days spent in the ICU by $4.8 \mathrm{~h},(b=-0.2, t[18729]=-3.0, p=0.002)$. To examine the impact of the number of PECs on total days spent in the hospital, we collapsed patients with PEC counts of 3 or more into one group with an assigned PEC count value of 3 . The total number of diagnosed PECs did not significantly impact the number of days patients with msTBI spent in the hospital, ( $F[3$, $\left.18727]=1.64, p=0.18, \eta^{2}<0.001\right)$.

Hypothesis 3: Examining how PECs combine to confer risk

Comparison of the four elbow plots generated by the various $\mathrm{K}$-modes clustering runs confirmed four as the optimal number of PEC profiles (Fig. 2). We subsequently named these clusters based on their predominant PEC categories (Fig. 4). The four clusters that emerged were: 1) Behavioral Risk, which includes patients with zero recorded PECs $(n=6387)$, and individuals whose primary diagnosed PECs were Substance Use Disorder and/or Current Smoker ( $n=5087) ; 2)$ Psychiatric and Substance Use Risk; 3) Cardiovascular Risk; and 4) Elevated Cardiovascular and Neuropsychiatric Risk, which consists of patients with a combination of three or more comorbid PEC diagnoses. Additional information regarding characteristic PEC sub-categories and demographics of these clusters is provided in Table 2 .

Exploratory question 1: Characteristics of patients with increasing quantities of PECs

Examination of PEC counts (patients with msTBI reporting 0,1 to 2 , or 3 or more PECs) regardless of 


\section{Pre-existing Conditions (PECs) Across All Patients $(\mathrm{N}=\mathbf{2 5 7 , 0 8 9})$}
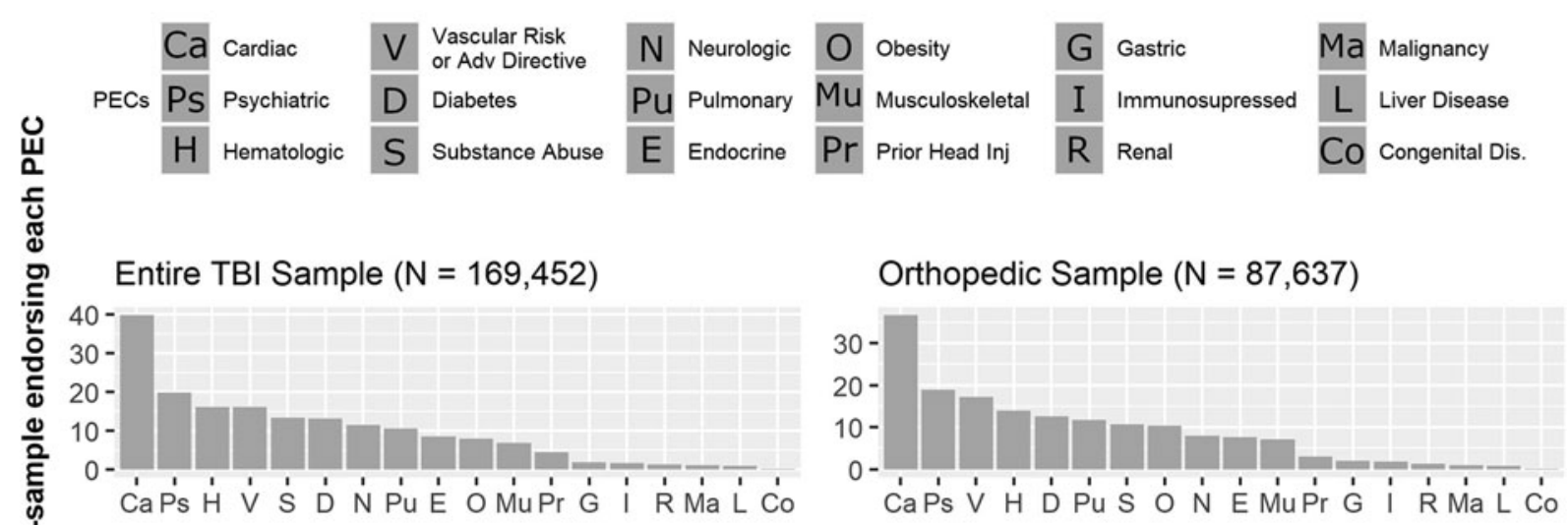

Mild TBI Sample $(N=150,721)$

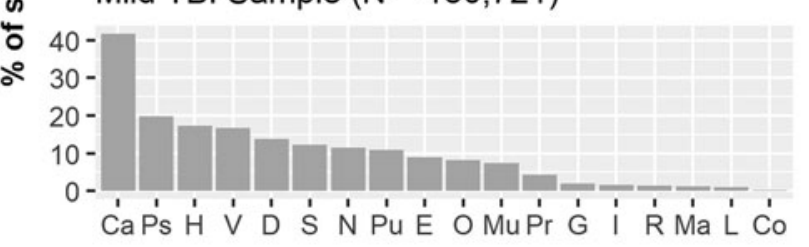

Moderate \& Severe TBI Sample $(\mathrm{N}=18,731)$

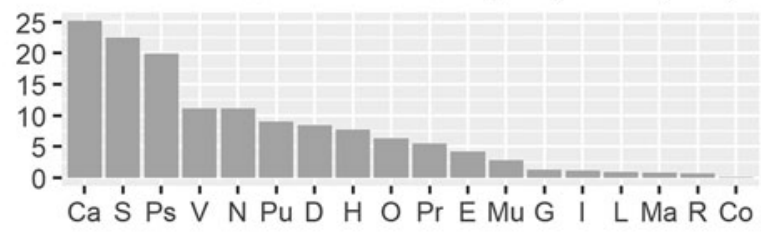

PECs

FIG. 3. Relative frequency of distinct PECs. Note frequencies for the Entire TBI Sample, Mild TBI Sample, and Orthopedic Sample remain similar. The Moderate and Severe TBI Sample reveals higher frequencies for substance use ( 1 in approximately 4) compared with the other three frequency graphs ( 1 in approximately 7). $\mathrm{PEC}$, pre-existing condition; $\mathrm{TBI}$, traumatic brain injury.

cluster membership reveals descriptive differences across the three groups with respect to demographics, but not hospital outcomes variables. Increasing PEC counts are positively associated with age and the proportion of female patients, but not with increasing stays in the ICU or total hospital days. However, the average hospital lengths of stay and days spent in the ICU were approximately 14 total days in the hospital and around 8 days in the ICU for all three PEC count groups.

\section{Exploratory question 2: Frequency of PECs} within TBI and orthopedic injury

Comparison of the entire TBI sample with the orthopedic injury sample reveals similar frequencies of PEC categories across both groups, supporting the decision to include all patients regardless of injury status in the $\mathrm{K}$-modes clustering algorithm. The four most frequently reported PECs categories for both orthopedic patients and patients with mTBI were cardiac, psychiatric, hematologic, and vascular risk (predominantly smoking) conditions. These same frequencies, however, did not hold for patients with msTBI, with cardiac, substance use, psychiatric, vascular risk (again predominantly smoking), and neurological conditions reported in the msTBI group.

\section{Exploratory question 3: Change in PECs}

over the lifespan

K-modes results demonstrate a clear demographic shift in the qualitative and quantitative characteristics (type and count) of the PEC clusters within msTBI (see Fig. 5). More specifically, the Behavioral Risk and Psychiatric and Substance Use Risk PEC profile groups are both younger on average than the Cardiovascular Risk and Elevated Cardiovascular and Neuropsychiatric Risk clusters. Shifts in the emergence of PEC cluster types are most evident when patients are in their fifties 


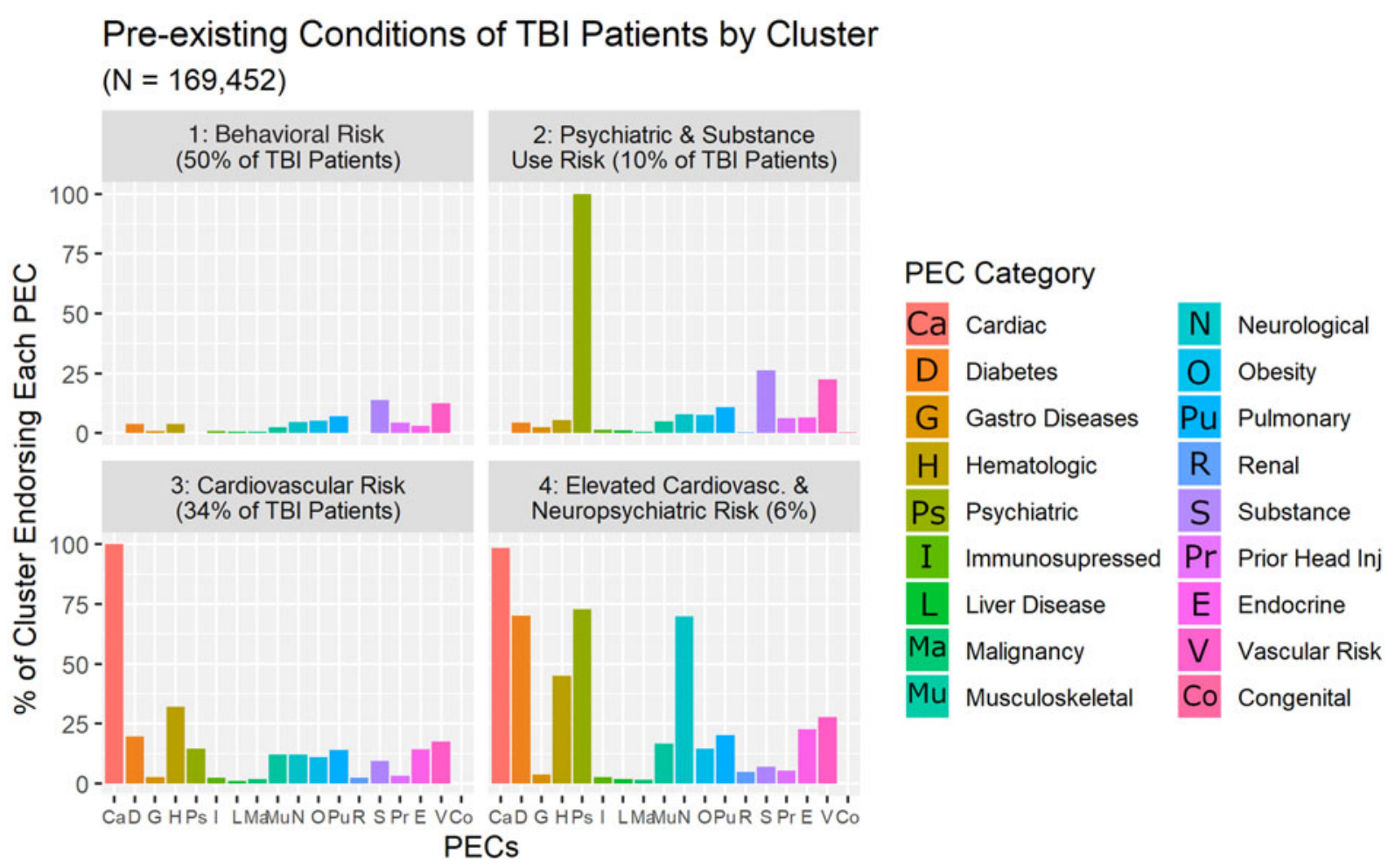

FIG. 4. Results of the K-modes cluster analysis. Four clusters are represented in panels: 1) Behavioral Risk: which includes patients with no recorded PECs, and individuals whose primary diagnosed PECs were Substance Use Disorder and/or Current Smoker; 2) Psychiatric and Substance Use Risk: primary PEC diagnoses were Psychological or Personality Disorder, followed by Substance Use Disorder, and Current Smoker; 3) Cardiovascular Risk: patients with predominantly Cardiac Conditions, followed by Hematologic, Diabetes, and Vascular Risk PECs; 4) Elevated Cardiovascular and Neuropsychiatric Risk: patients primarily with Cardiac, Psychiatric, Diabetes, Neurological, and Vascular Risk conditions. PEC, pre-existing condition; $\mathrm{TBI}$, traumatic brain injury.

and sixties, but emerge as early as one's forties (Cardiovascular Risk cluster), and persist in to one's seventies (Elevated Cardiovascular + Neuropsychiatric Risk). Further, as the mean age of the PEC clusters begins to increase, so too do the proportions of female patients represented within each cluster.

To ensure equal cell sizes for the one-way analysis of variance (ANOVA) tests (Welch's $F$ ), we randomly sampled 500 cases per cluster from the total subsample of patients with msTBI $(n=18,731)$. Results revealed cluster group differences in FIM ( $F[3$, $1108.5]=51.4, p<0.001)$ and lengths of stay both in the ICU $(F[3,1105.5]=7.3, p<0.001)$ as well as the hospital overall $(F[3,1099.9]=5.7, p<0.001)$. Post hoc tests with Bonferroni correction revealed mean differences in FIM scores between the Behavioral Risk cluster $(\bar{M}=14.9)$ and the two oldest clusters: Cardiovascular Risk $(\bar{M}=13.0$, Cohen's d=0.4) $)$ and Elevated Cardiovascular and Neuropsychiatric Risk $(\bar{M}=12.1$, Cohen's $\mathrm{d}=0.6$; both $p<0.001)$, but no significant differences in mean FIM scores when compared with the Psychiatric and Substance Use Risk cluster $(\bar{M}=15.6, p=0.3))$. Similarly, the Psychiatric and Substance Use Risk cluster mean FIM score was also significantly higher than the two oldest clusters (both $p<0.001$ ). Finally, mean FIM scores did not differ significantly between the Cardiovascular Risk and Elevated Cardiovascular and Neuropsychiatric Risk, $p=0.08$.

With respect to total days in the hospital, mean differences did not reach significance for comparisons across the Behavioral Risk ( $\bar{M}=14.2$ days $)$, Psychiatric 
Table 2. Descriptive Characteristics by K-Modes Cluster of Patients with Moderate and Severe TBI

\begin{tabular}{lcccc}
\hline Cluster (n) & $\begin{array}{c}\bar{M} \text { age }(S D) \\
\text { in years }\end{array}$ & Male & $\begin{array}{c}\text { Caucasian }^{a} \\
\text { (African-American) }\end{array}$ & $\begin{array}{c}\bar{M} \text { Median hospital days score } \\
\text { on admission }\end{array}$ \\
\hline
\end{tabular}

Behavioral Risk (total $n=11,474$ ), consisting of two subgroups

1. No PECs reported $(6,387,56 \%)$

$33(14.3)$

$78 \%$

$83 \%(11 \%)$

10

5.6

$\%$ of group endorsing trait $\%$ only endorsing this trait

\begin{tabular}{llllc}
\cline { 2 - 4 } No PECs reported & \multicolumn{2}{c}{$100 \%$} & & $100 \%$ \\
\hline 2. PEC-positive $(5087,44 \%)$ & $41(17.4)$ & $77 \%$ & $81 \%(15 \%)$ & 9 \\
\hline
\end{tabular}

\begin{tabular}{|c|c|c|c|c|}
\hline \multirow{5}{*}{$\begin{array}{l}\text { Chronic alcohol abuse } \\
\text { Substance use disorder } \\
\text { Psychiatric and Substance Use Risk (2523) } \\
\text { PEC sub-categories }\end{array}$} & \multirow{2}{*}{$\frac{\% \text { of group endorsing trait }}{35 \%}$} & \multicolumn{3}{|c|}{$\%$ only endorsing this trait } \\
\hline & & \multicolumn{3}{|c|}{$23 \%$} \\
\hline & $22 \%$ & \multicolumn{3}{|c|}{$15 \%$} \\
\hline & $39(15.9) \quad 65 \%$ & $89 \%(9 \%)$ & 10 & 6.1 \\
\hline & $\%$ of cluster endorsing trait & \multicolumn{3}{|c|}{$\%$ only endorsing this trait } \\
\hline Diagnosed psychiatric/Personality disorder & $91 \%$ & \multicolumn{3}{|c|}{$31 \%$} \\
\hline Chronic alcohol abuse & $25 \%$ & \multicolumn{3}{|c|}{$0 \%$} \\
\hline Substance use disorder & $19 \%$ & \multicolumn{3}{|c|}{$0 \%$} \\
\hline Current smoker & $14 \%$ & \multicolumn{3}{|c|}{$0 \%$} \\
\hline Diagnosed ADHD & $10 \%$ & \multirow{2}{*}{\multicolumn{3}{|c|}{$\begin{array}{l}4 \% \\
0 \%\end{array}$}} \\
\hline Respiratory disease or COPD & $10 \%$ & & & $0 \%$ \\
\hline Cardiovascular Risk (3903) & $64(17.2) \quad 67 \%$ & $87 \%(11 \%)$ & 11 & 6.8 \\
\hline PEC sub-categories & $\%$ of cluster endorsing trait & \multicolumn{3}{|c|}{$\%$ only endorsing this trait } \\
\hline Hypertension requiring medication & $89 \%$ & \multicolumn{3}{|c|}{$18 \%$} \\
\hline Coronary artery disease & $22 \%$ & \multicolumn{3}{|c|}{$3 \%$} \\
\hline Chronic alcohol abuse & $17 \%$ & \multicolumn{3}{|c|}{$0 \%$} \\
\hline Diabetes mellitus & $16 \%$ & & $0 \%$ & \\
\hline Diagnosed psychiatric/Personality disorder & $15 \%$ & & $0 \%$ & \\
\hline Obesity & $10 \%$ & & $0 \%$ & \\
\hline Respiratory disease or COPD & $14 \%$ & & $0 \%$ & \\
\hline Elevated Cardiovascular and Neuropsychiatric Risk (831) & $72(15.7) \quad 50 \%$ & $89 \%(9 \%)$ & 7 & 8.3 \\
\hline PEC sub-categories & $\%$ of cluster endorsing trait & & $\%$ only endorsing this trait & \\
\hline Hypertension requiring medication & $87 \%$ & & $0 \%$ & \\
\hline Diagnosed psychiatric/Personality disorder & $71 \%$ & & $0 \%$ & \\
\hline Diabetes mellitus & $66 \%$ & & $0 \%$ & \\
\hline Dementia & $49 \%^{\mathrm{c}}$ & & $0 \%$ & \\
\hline CVA/Hemiparesis (stroke with residual) & $31 \%$ & & $0 \%$ & \\
\hline Coronary artery disease & $33 \%$ & & $0 \%$ & \\
\hline Anticoagulant therapy & $29 \%^{d}$ & & $0 \%$ & \\
\hline Respiratory disease or COPD & $19 \%$ & & $0 \%$ & \\
\hline Seizures & $14 \%$ & & $0 \%$ & \\
\hline Functionally dependent health status & $14 \%$ & & $0 \%$ & \\
\hline Congestive heart failure & $14 \%$ & & $0 \%$ & \\
\hline Arthritis & $11 \%$ & & $0 \%$ & \\
\hline Obesity & $11 \%$ & & $0 \%$ & \\
\hline Chronic alcohol abuse & $11 \%$ & & $0 \%$ & \\
\hline History of cardiac surgery & $10.5 \%$ & & $0 \%$ & \\
\hline Advanced directive-limited care & $10 \%$ & & $0 \%$ & \\
\hline
\end{tabular}

${ }^{\mathrm{a}}$ Race data were not available for all participants.

${ }^{\mathrm{b}} \mathrm{PEC}$ sub-categories were those endorsed by at least $10 \%$ of the cluster.

'Coding changes over the lifetime of the PTOS database collapsed previously separate codes for 1. Alzheimer's disease and 2. chronic dementia, into a new third code for Dementia. The percentage in this table is the sum of those three subcategories.

${ }^{\mathrm{d} C}$ Coding collapsed previously separate codes for Anticoagulant therapy, Anti-platelet agents, and Pradaxa therapy in to one new code for Anticoagulant therapy. The percentage in this table is the sum of those four subcategories.

ADHD, attention deficit hyperactivity disorder; COPD, chronic obstructive pulmonary disease; CVD, cardiovascular disease; GCS, Glasgow Coma Scale; PEC, pre-exisiting health condition; PTOS, Pennsylvania Trauma Outcomes Study; SD, standard deviation; TBI, traumatic brain injury. 


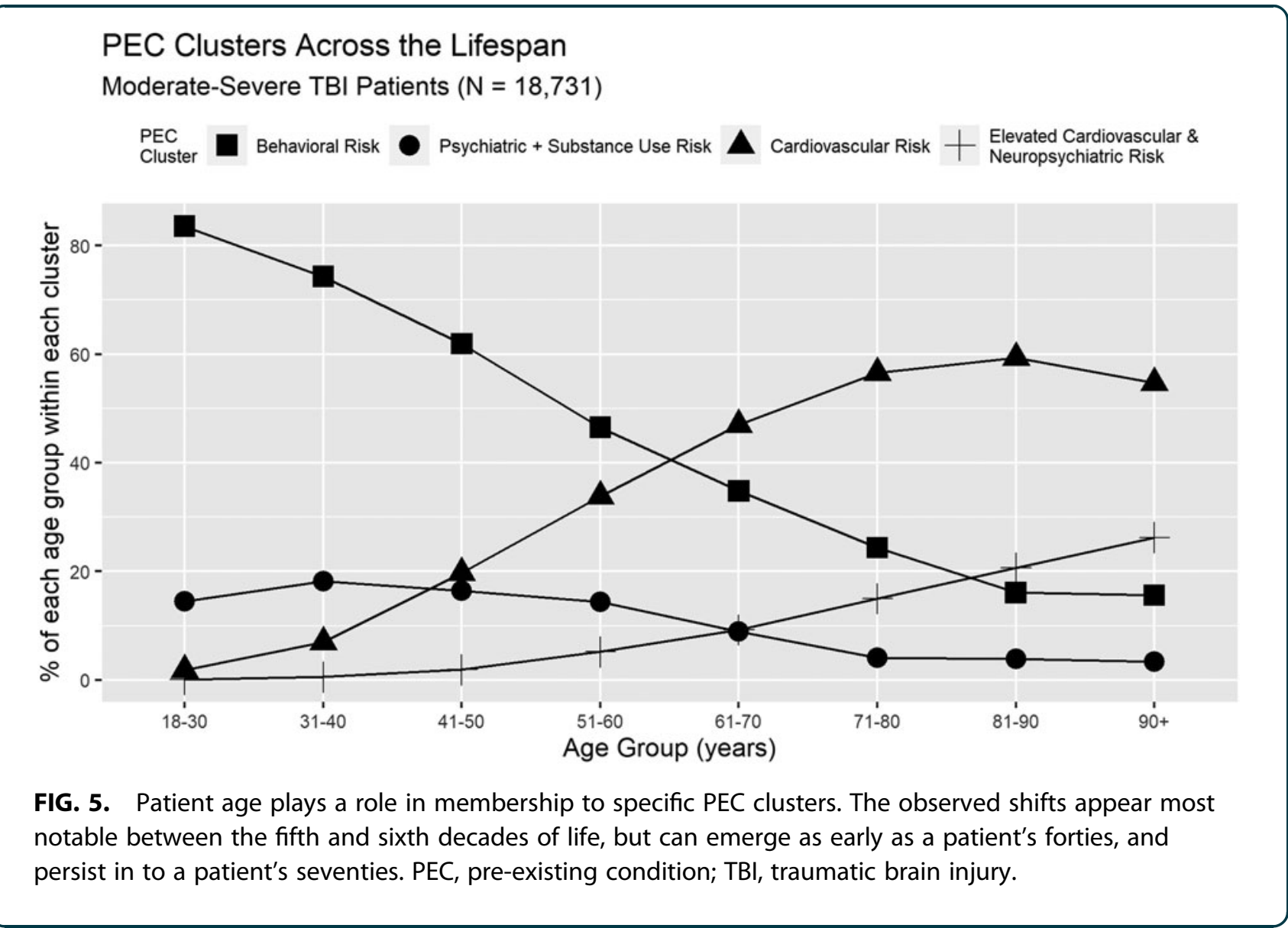

and Substance Use Risk $(\bar{M}=14.5$ days $)$, and Cardiovascular Risk clusters $(\bar{M}=13.8$ days; all $p>0.05)$, but did so for each of these clusters when compared with the Elevated Cardiovascular and Neuropsychiatric Risk cluster $(\bar{M}=11.3$ days, $p$-values $=0.02,0.005$, and 0.05 , respectively, all Cohen's d sizes $=0.2$ ). Finally, although days in the ICU for the two younger groups (Behavioral Risk $[\bar{M}=8.3$ days $]$ and Psychiatric and Substance Use Risk $[\bar{M}=7.5$ days $]$ ) do not differ significantly from one another $(p=1.0)$ or from the Cardiovascular Risk cluster $(\bar{M}=8.5$ days, $p$-value $=1.0$, $p$-value $=0.7)$; patients in all three clusters spent more time in the ICU in comparison with the Elevated Cardiovascular and Neuropsychiatric Risk cluster $(\bar{M}=5.8$ days, $p$ - values $<0.001,0.04$, and $<0.001$, respectively, all Cohen's d sizes $=0.2$ ).

\section{Discussion}

We aimed to understand relative frequency of distinct PECs after trauma and the influence PECs have for mortality and early outcomes indicators for msTBI.
We leveraged the enormous resources in the PTOS to conduct a population-based study of PECs in TBI. Clusters of clinically relevant PECs can be reliably observed in trauma populations, and hold important implications for early patient outcomes. The data also reveal that the frequency for both simple PEC count (i.e., number of PECs any one patient may have) and PEC clusters (symptom groupings) are critically influenced by age and even injury severity, but their respective effects on post-injury outcomes may emerge months to years following hospital discharge, and thus are not captured in these hospital admission data.

\section{Impact of PECs on acute TBI recovery trajectories}

Within the patients with msTBI who were examined, the sheer number and types of PECs had consequence for outcomes. First, as the number of PECs increases, mortality rate climbs incrementally by a factor of 1.7-1.9. Second, distinct PEC cluster types confer differential risk for recovery following injury, and perhaps unsurprisingly, age plays a critical role in the 
emergence of PECs (Fig. 5). In the early recovery period, these PEC clusters also confer risk of differential recovery following msTBI as measured by FIM, days spent in the hospital, and days in the ICU. The impact of PECs on recovery from TBI appears linked to the interactive characteristics of the chronic conditions carried by the patients with msTBI examined here, rather than the overall total number of PECs as evidenced by the small effect sizes associated with our results.

Age and PECs hold association with injury severity Given the preponderance of mTBI injuries (more than $60 \%$ ) across all four clusters in the initial K-modes clustering results, we also suggest these data may speak to differences in injury mechanism as a result of both age and PEC types. ${ }^{65}$ More specifically, although the younger PEC profiles present with lower total number of PEC sub-categories, they also have a greater proportion of severe TBI cases in comparison with the two older PEC profiles, representing a trade-off between decreasing injury severity with age, and increased aggregation of age-dependent PECs. Further, only $6 \%$ or fewer of patients with TBI endorsed one PEC exclusively. More specifically, it appears that PEC diagnoses frequently co-occur, and this comorbidity does not appear restricted solely to a function of advanced age.

\section{Influence of sex on PECs}

Patient sex proportions also appear to shift within the PEC clusters. Although the Behavioral Risk cluster contains the largest proportion of injured males, the Psychiatric and Substance Use Risk cluster demonstrates an increased number of injured females despite being of similar mean age and proportions of TBI severity. A key sex-by-PEC sub-category difference between these two clusters is the Psychiatric/Personality Disorder diagnosis; $43 \%$ of patients within this cluster endorsing this trait were female. Within the Cardiovascular Risk and Elevated Cardiovascular Risk clusters, an increase in mean age also emerges alongside a switch from a greater number of males to more female injuries, and an increase in the total number of reported PECs, aligning with prior research in both TBI and aging. ${ }^{38}$

\section{Inflammation and immunosenescence}

as part of $T B I$ research

Accumulation of PECs induces necessary inflammatory processes in the acute phase; however, prolonged exposure to sub-clinical hyper-inflammation resulting from chronic conditions compromises the body's ability to effectively respond to additional physical and neurological insults. ${ }^{66-69}$ Despite established research examining reciprocal impacts between central and peripheral inflammatory processes, ${ }^{19,41,42,70,71}$ research protocols frequently detail omission of potential participants with diagnosed PECs, notably psychiatric conditions. As global populations continue to age, understanding the impact of multiple morbidity, and the interactions between timing and type of PEC onset, notably with advancing age, remain critical avenues for research determining both risk of and recovery from injuries. ${ }^{30,72,73}$

More specifically, overly restrictive exclusion criteria can eliminate the variability imparted by the presence of PECs, and impact understanding of health mechanisms that influence outcomes trajectory. ${ }^{31,38,74,75}$ For example, Isokourtti and colleagues ${ }^{74}$ demonstrated that in a Finnish cohort of patients with mTBI $(n=3023)$, only $2.5 \%$ (76 patients) met criteria for isolated mTBI, that is a patient with mTBI absent of any pre-existing medical or mental health problems. The broad PEC categories described by Isokourtti and colleagues $^{74}$ are consistent with both our findings here and mirror research in TBI and non-TBI patient cohorts across the lifespan. ${ }^{15,30,31,35,76,77}$ Patients with msTBI presenting with no diagnosed PECs comprise only about $25 \%$ of the TBI sample analyzed here, so continued focus on "isolated TBI" samples for research protocols will not only exclude a majority of affected patients with TBI (thus leading to poor generalizability of findings), but also eliminate opportunities to examine the comorbidities complicating patient recovery and the associated costs. Further, TBI research is under-served by the under-representation of geriatric patient samples, ${ }^{38,78-80}$ often due to restrictive inclusion criteria. ${ }^{38,81,82}$ This poses significant challenges to understanding post-injury outcomes that may emerge years to decades post-injury. ${ }^{83,84}$ This is particularly concerning, given the increase in TBI-related hospitalizations and deaths in geriatric populations in comparison with other age demographics over the last several years. $38,65,78,80,81$

Our findings align with a growing literature examining the impact of pre-existing health conditions on recovery following TBI. More specifically, Kumar and colleagues ${ }^{15}$ implemented Treelet Transform classification, which resulted in three clusters, which they broadly classified as "acute medical diseases/infections, 
chronic conditions, and substance abuse disorders." Similarities between our results and the Treelet Transform clusters offered by Kumar and colleagues include the emergence of chronic conditions, specifically those impacting cardiovascular health, such as cardiac conditions (including heart disease) and diabetes. Additional commonalities include the emergence of substance use disorders, a prominent feature in two of our four clusters. Two important distinctions between our classification methods, however, warrant mention. First, our resulting clusters from K-modes clustering do not permit patients to belong to more than one cluster, whereas Treelet Transform clustering undertaken by Kumar and colleagues ${ }^{27}$ does permit patients to have overlapping cluster membership. Second, the PTOS defines PECs as conditions present before admission to the hospital, and thereby does not code for hospitalacquired infections (nosocomial conditions); this is in contrast to the data analyzed by Kumar and colleagues, ${ }^{15}$ which included hospital codes documenting health conditions present prior to hospital entry and those acquired during patients' hospital stays.

Further, one of our overarching research aims of descriptively examining all PECs rather than focusing on an individual or subset of conditions aligns with yet additional work by Kumar and colleagues. ${ }^{28}$ Although our work presented here stems from trauma center data in the acute stage post-injury, and Kumar and colleagues ${ }^{28}$ examined outcomes 10 years post-injury, our findings speak to the importance of studying multimorbidity as it better simulates real-world conditions. Further, Kumar and colleagues ${ }^{28}$ also argue that physical and mental health conditions may reciprocally exacerbate one another, a qualitative aspect that is illustrated within our K-modes clustering results, and is highlighted in the discussion offered in this article regarding exclusion criteria that prohibit individuals with these conditions from enrolling in research.

\section{Limitations and future directions}

The results and proposed clinical implications should be considered in the context of the following caveats. The hospital-based data analyzed here may be impacted by an inherent selection bias of individuals who are more likely to seek medical services. Similarly, the de-identified nature of the data prevented assessment of whether the cases analyzed here were repetitive admissions for trauma. Repetitive experiences of TBI, and both neurological and psychiatric conditions have been demonstrated to confer greater risk of hospital re-entry for TBI. ${ }^{29}$ Additionally, given the emergency/trauma center environment, the cases analyzed here do not have pre-injury functional status data available to assess change in functional status from before hospital admission. Further, the cross-sectional data here do not delineate when patients were diagnosed with PECs, precluding our ability establish direct causality of specific PECs on either chronological acquisition of systemically related PECs or injury specifically. ${ }^{83}$

Relatedly, the availability of insurance (and access to care) may also impact whether PECs can be diagnosed, subsequently disclosed by these patients when they present for care, and may impact inclusion in research examining the questions presented here. The PTOS data permit coding for an array of insurance types, ${ }^{60}$ ranging from those without insurance coverage to those with a private indemnity, and the data presented here are from patients from every possible category of insurance $(6.8 \%$ did not identify a third-party payor and bills for service were rendered to the patient, compared with $7.9 \%$ prior to data cleaning). As a final point, prior work in our lab demonstrates that these differences in insurance exert influence on postdischarge outcomes, rather than the outcomes linked to acute hospital stay presented here. ${ }^{85}$

Further, the proportion of African-American patients without recorded PEC data, and the degree to which Hispanic ethnicity was missing from the data make it difficult to generalize these analyses to non-Caucasian patient populations. This data gap remains both concerning and critically important, considering current statistics that nonCaucasian patients are more likely to suffer from a greater number of PECs, have a higher incidence of TBI, and are at greater risk for developing dementia-related conditions (for which both prior TBI and pre-existing conditions confer greater risk). ${ }^{86}$ Given the known impact of race and ethnicity status on TBI outcomes, neurotrauma investigators should be proactive about how to guarantee that aggregated data are representative of brain injury as it occurs in the population, and that includes all race and socioeconomic demographics.

\section{Conclusion}

The findings presented across the aims of this study demonstrate that implementing a data-driven approach revealed distinct groups (clusters) of PECs across the lifespan. The prominent PECs present in these four clusters emerge at specific ages, with increasing age associated with a simultaneous increase in the quantity of specific age-related conditions, but also decreasing TBI 
severity. Similarly, PEC quantity and type were significantly, yet weakly associated with measures of functional independence and hospital stay. These findings add to the literature examining the impact of pre-existing conditions on risk of and recovery from TBI, and infer the importance of re-conceptualizing the impact of PECs on recovery above and beyond a single number or solitary condition. Continued inclusion of individuals absent any diagnosed PECs in TBI research captures only a limited scope of the population, indirectly omitting older and non-Caucasian patients with TBI who may be at greater risk for exacerbated recovery or pathological aging, and presents salient challenges to understanding recovery mechanisms post-TBI as well as for reproducibility of findings.

\section{Acknowledgments}

The data presented here were provided by the Pennsylvania Trauma Systems Foundation, Mechanicsburg, Pennsylvania (PTSF). The PTSF specifically disclaims responsibility for any analyses, interpretations, or conclusions. Credit must be given to the Pennsylvania Trauma Outcome Study (PTOS) as the source of the data, and we specifically thank Nathan McWilliams for his assistance in receiving these data.

This study was pre-registered with the Center for Open Science-Open Science Framework (OSF).

\section{Authors' Contributions}

K.D. contributed to project conceptualization, data cleaning, analyses, and drafting of the manuscript; E.G. assisted with data cleaning and drafting the manuscript, J.S. contributed to data analyses, data cleaning, and drafting the manuscript; P.S. contributed to data cleaning and drafting the manuscript; and F.H. assisted with project conceptualization, drafting the manuscript, and supervision of the project.

\section{Funding Information}

This project is funded, in part, under a Grant with the Pennsylvania Department of Health (PA-DOH SAP\# 4100077082). The Department specifically disclaims responsibility for any analyses, interpretations or conclusions.

\section{Author Disclosure Statement}

No competing financial interests exist.

\section{Supplementary Material}

Supplementary Table S1

\section{References}

1. Iverson, G.L., Gardner, A.J., Terry, D.P., Ponsford, J.L., Sills, A.K., Broshek, D.K., and Solomon, G.S. (2017). Predictors of clinical recovery from concussion: a systematic review. Br. J. Sports Med. 51, 941-948.

2. Shetty, T., Nguyen, J.T., Cogsil, T., Tsiouris, A.J., Niogi, S.N., Kim, E.U., Dalal, A., Halvorsen, K., Cummings, K., Zhang, T., Masdeu, J.C., Mukherjee, P., and Marinelli, L. (2018). Clinical findings in a multicenter MRI study of mild TBI. Front. Neurol. 9, 1-10.

3. Edwards, K.A., Pattinson, C.L., Guedes, V.A., Peyer, J., Moore, C., Davis, T., Devoto, C., Turtzo, L.C., Latour, L., and Gill, J.M. (2020). Inflammatory cytokines associate with neuroimaging after acute mild traumatic brain injury. Front. Neurol. 11, 1-7.

4. Greco, T., Ferguson, L., Giza, C., and Prins, M.L. (2019). Mechanisms underlying vulnerabilities after repeat mild traumatic brain injuries. Exp. Neurol. 317, 206-213.

5. Schneider, E.B., Sur, S., Raymont, V., Duckworth, J., Kowalski, R.G., Efron, D.T., Hui, X., Selvarajah, S., Hambridge, H.L., and Stevens, R.D. (2014). Functional recovery after moderate/severe traumatic brain injury: a role for cognitive reserve? Neurology 82, 1636-1642.

6. Niemeier, J.P., Perrin, P.B., Holcomb, M.G., Rolston, C.D., Artman, L.K., Lu, J., and Nersessova, K.S. (2014). Gender differences in awareness and outcomes during acute traumatic brain injury recovery. J. Women's Health (Larchmt) 23, 573-580.

7. Hayes, J.P., Bigler, E.D., Verfaellie, M., and City, S.L. (2016). Traumatic brain injury as a disorder of brain connectivity. J. Int. Neuropsychol. Soc. 22, 120-137.

8. Ware, J.B., Dolui, S., Duda, J., Gaggi, N., Choi, R., Detre, J., Whyte, J., DiazArrastia, R., and Kim, J.J. (2020). Relationship of cerebral blood flow to cognitive function and recovery in early chronic traumatic brain injury. J. Neurotrauma 2187, 2180-2187.

9. Marklund, N., Bellander, B., Godbolt, A., Levin, H., McCrory, P., and Thelin, E. (2019). Treatments and rehabilitation in acute and chronic state of traumatic brain injury. J. Intern. Med. 285, 608-623.

10. Zahniser, E., Nelson, L.D., Dikmen, S.S., Machamer, J.E., Stein, M.B., Yuh, E., Manley, G.T., and Temkin, N.R. (2019). The temporal relationship of mental health problems and functional limitations following mTBI: a TRACK-TBI and TED study. J. Neurotrauma 36, 1786-1793.

11. Rabinowitz, A.R., Hart, T., Whyte, J., and Kim, J. (2018). Neuropsychological recovery trajectories in moderate to severe traumatic brain injury: influence of patient characteristics and diffuse axonal injury-erratum. J. Int. Neuropsychol. Soc. 24, 237-246.

12. Yeates, K.O., Levin, H.S., and Ponsford, J. (2017). The neuropsychology of traumatic brain injury: looking back, peering ahead. J. Int. Neuropsychol. Soc. 23, 806-817.

13. Albrecht, J.S., Abariga, S.A., Rao, V., and Wickwire, E.M. (2020). Incidence of new neuropsychiatric disorder diagnoses following traumatic brain injury. J. Head Trauma Rehabil. 35, E352-E360.

14. Beaulieu-Bonneau, S., St-Onge, F., Blackburn, M.C., Banville, A., ParadisGiroux, A.A., and Ouellet, M.C. (2018). Alcohol and drug use before and during the first year after traumatic brain injury. J. Head Trauma Rehabil. 33, E51-E60.

15. Kumar, R.G., Juengst, S.B., Wang, Z., Dams-O'Connor, K., Dikmen, S.S. O'Neil-Pirozzi, T.M., Dahdah, M.N., Hammond, F.M., Felix, E.R., Arenth, P.M., and Wagner, A.K. (2018). Epidemiology of comorbid conditions among adults 50 years and older with traumatic brain injury. J. Head Trauma Rehabil. 33, 15-24.

16. Yue, J.K., Cnossen, M.C., Winkler, E.A., Deng, H., Phelps, R.R.L., Coss, N.A., Sharma, S., Robinson, C.K., Suen, C.G., Vassar, M.J., Schnyer, D.M., Puccio, A.M., Gardner, R.C., Yuh, E.L., Mukherjee, P., Valadka, A.B., Okonkwo, D.O. Lingsma, H.F., Manley, G.T., Cooper, S.R., Dams-O'Connor, K., Gordon, W.A. Hricik, A.J., Maas, A.I.R., Menon, D.K., and Morabito, D.J. (2019). Pre-injury comorbidities are associated with functional impairment and postconcussive symptoms at 3- and 6-months after mild traumatic brain injury: a Track-TBI study. Front. Neurol. 10, 343.

17. Kesinger, M.R., Kumar, R.G., K,W.A., Puyana, J.C., Peitzman, A.P., Billiar, T.R., and Sperry, J.L. (2015). Hospital acquired pneumonia is an independent predictor of poor global outcome in severe TBI up to 5 years after discharge. J. Trauma Acute Care Surg. 78, 396-402.

18. Kumar, R.G., Kesinger, M.R., Juengst, S.B., Brooks, M.M., Fabio, A., Dams-O'connor, K., Pugh, M.J., Sperry, J.L., and Wagner, A.K. (2020). Effects of hospital-acquired pneumonia on long-term recovery and hospital 
resource utilization following moderate to severe traumatic brain injury. J. Trauma Acute Care Surg. 88, 491-500.

19. Sharma, R., Shultz, S.R., Robinson, M.J., Belli, A., Hibbs, M.L., O'Brien, T.J., and Semple, B.D. (2019). Infections after a traumatic brain injury: the complex interplay between the immune and neurological systems. Brain Behav. Immun. 79, 63-74.

20. Albrecht, J.S., Lydecker, A., Peters, M., and Rao, V. (2020). Treatment of depression following traumatic brain injury reduces risk of neuropsychiatric outcomes. J. Neurotrauma 37, 1-7.

21. Bertisch, H., Krellman, J.W., Bergquist, T.F., Dreer, L.E., Ellois, V., and Bushnik, T. (2017). Characteristics of firearm brain injury survivors in the Traumatic Brain Injury Model Systems (TBIMS) national database: a comparison of assault and self-inflicted injury survivors. Arch. Phys. Med Rehabil. 98, 2288-2294.

22. Bombardier, C.H., Hoekstra, T., Dikmen, S., and Fann, J.R. (2016). Depression trajectories during the first year after traumatic brain injury. J. Neurotrauma $33,2115-2124$.

23. Unsworth, D.J., and Mathias, J.L. (2017). Traumatic brain injury and alcohol/substance abuse: a Bayesian meta-analysis comparing the outcomes of people with and without a history of abuse. J. Clin. Exp. Neuropsychol. 39, 547-562.

24. Malec, J., Ketchum, J.M., Hammond, F.M., Corrigan, J.D., Dams-O'Connor K., Hart, T., Novack, T., Dahdah, M., Whiteneck, G.G., and Bogner, J. (2019) Longitudinal effects of medical comorbidities on functional outcome and life satisfaction after traumatic brain injury: an individual growth curve analysis of NIDILIRR Traumatic Brain Injury Model Systems Data. J. Head Trauma Rehabil. 34, E24-E35.

25. Centers for Disease Control and Prevention. (2020). About Chronic Diseases. National Center for Chronic Disease Prevention and Health Promotion. https://www.cdc.gov/chronicdisease/about/index.htm (Last accessed September 29, 2020).

26. Buttorff, C., Ruder, T., and Bauman, M. (2017). Multiple chronic conditions in the United States. RAND Corporation: Santa Monica, CA.

27. Kumar, R.G., Olsen, J., Juengst, S.B., Dams-O'Connor, K., O'Neil-Pirozzi, T.M., Hammond, F.M., and Wagner, A.K. (2019). Comorbid conditions among adults 50 years and older with traumatic brain injury: examining associations with demographics, healthcare utilization, institutionalization, and 1-year outcomes. J. Head Trauma Rehabil. 34, 224-232.

28. Kumar, R.G., Ketchum, J.M., Corrigan, J.D., Hammond, F.M., Sevigny, M., and Dams-O'Connor, K. (2020). The longitudinal effects of comorbid health burden on functional outcomes for adults with moderate to severe traumatic brain injury. J. Head Trauma Rehabil. 35, E372-E381.

29. Hayward, R.D., Fessler, M.M., Buck, J., and Fessler, R.D. (2018). Risk factors for recurrent neurotrauma: a population-based study in Southeastern Michigan. Brain Inj. 32, 1373-1376.

30. Dams-O'Connor, K., Gibbons, L., Landau, A., Larson, E., and Crane, P. (2016). Health problems precede traumatic brain injury in older adults. J. Am. Geriatr. Soc. 64, 844-848.

31. Calvo, R.Y., Lindsay, S.P., Edland, S.D., Macera, C.A., Wingard, D.L., OhnoMachado, L., and Sise, M.J. (2016). The differential associations of preexisting conditions with trauma-related outcomes in the presence of competing risks. Injury 47, 677-684.

32. Kuhne, C.A., Ruchholtz, S., Kaiser, G.M., Nast-Kolb, D., Bouillon, B., Eich, S., Kanz, K.G., Kühne, C.A., Lackner, C., Lefering, R., Lewan, U., Mutschler, W. Nast-Kolb, D., Neugebauer, E., Oestern, H.J., Paffrath, T., Pape, H.C., Pirente, N., Raum, M., Rieger, G., Rixen, D., Ruchholtz, S., Schlosser, L., Strohecker, C., Waydhas, C., and Zintl, B. (2005). Mortality in severely injured elderly trauma patients: when does age become a risk factor? World J. Surg. 29, 1476-1482.

33. Brown, K., Cameron, I.D., Keay, L., Coxon, K., and Ivers, R. (2017). Functioning and health-related quality of life following injury in older people: a systematic review. Inj. Prev. 23, 403-411.

34. Sammy, I., Lecky, F., Sutton, A., Leaviss, J., and O'Cathain, A. (2016). Factors affecting mortality in older trauma patients: a systematic review and meta-analysis. Injury 47, 1170-1183.

35. Chan, V., Mollayeva, T., Ottenbacher, K.J., and Colantonio, A. (2017). Clinical profile and comorbidity of traumatic brain injury among younger and older men and women: a brief research notes. BMC Res. Notes 10 , $1-7$.

36. Hamel, R.N., and Smoliga, J.M. (2019). Physical activity intolerance and cardiorespiratory dysfunction in patients with moderate-to-severe traumatic brain injury. Sport Med. 49, 1183-1198.
37. Wilson, L., Stewart, W., Dams-O'Connor, K., Diaz-Arrastia, R., Horton, L, Menon, D.K., and Polinder, S. (2017). The chronic and evolving neurological consequences of traumatic brain injury. Lancet Neurol. 16, 813-825.

38. Gardner, R.C., Dams-O'Connor, K., Morrissey, M.R., and Manley, G.T. (2018). Geriatric traumatic brain injury: epidemiology, outcomes, knowledge gaps, and future directions. J. Neurotrauma 35, 889-906.

39. Bobeff, E.J., Fortuniak, J., Bryszewski, B., Wiśniewski, K., Bryl, M., Kwiecień, K., Stawiski, K., and Jaskólski, D.J. (2019). Mortality after traumatic brain injury in elderly patients: a new scoring system. World Neurosurg. 128, e129-e147.

40. Hammond, F.M., Corrigan, J.D., Ketchum, J.M., Malec, J.F., Conner, K.D., Hart, T., Novack, T.A., Dahdah, M.N., and Whiteneck, G.G. (2020). Prevalence of medical and psychiatric comorbidities following traumatic brain injury. J. Head Trauma Rehabil. 34, E1-E10.

41. Needham, E.J., Helmy, A., Zanier, E.R., Jones, J.L., Coles, A.J., and Menon D.K. (2019). The immunological response to traumatic brain injury. J. Neuroimmunol. 332, 112-125.

42. Anthony, D.C., Couch, Y., Losey, P., and Evans, M.C. (2012). The systemic response to brain injury and disease. Brain Behav. Immun. 26, 534-540.

43. Miller, A.H., and Raison, C.L. (2017). Role of inflammation in depression. Nat. Rev. Immunol. 16, 22-34.

44. Dams-O'Connor, K., Pretz, C., Mellick, D., Dreer, L.E., Hammond, F.M. Hoffman, J., Landau, A., and Zafonte, R. (2017). Rehospitalization over 10 years among survivors of TBI: a National Institute on Disability, Independent Living and Rehabilitation Research (NIDILRR) Traumatic Brain Injury Model Systems Study. J. Head Trauma Rehabil. 32, 147-157.

45. Dams-O'Connor, K., Spielman, L., Singh, A., Gordon, W.A., Lingsma, H.F., Maas, A.I.R., Manley, G.T., Mukherjee, P., Okonkwo, D.O., Puccio, A.M., Schnyer, D.M., Valadka, A.B., Yue, J.K., Yuh, E.L., and the TRACK-TBI Investigators. (2013). The impact of previous traumatic brain injury on health and functioning: a TRACK-TBI study. J. Neurotrauma 30, 2014-2020.

46. Dams-O'Connor, K., Spielman, L., Hammond, F.M., Sayed, N., Culver, C., and Diaz-Arrastia, R. (2013). An exploration of clinical dementia phenotypes among individuals with and without traumatic brain injury. NeuroRehabilitation 32, 199-209.

47. Hillary, F.G., and Medaglia, J.D. (2020). What the replication crisis means for intervention science. Int. J. Psychophysiol. 154, 3-5.

48. Bannon, S.M., Kumar, R.G., Bogner, J., O'Neil-Pirozzi, T.M., Spielman, L., Watson, E.M., and Dams-O'Connor, K. (2020). Reinjury after moderate to severe TBI. J. Head Trauma Rehabil. 36, E50-E60.

49. Stein, D.G. (2015). Embracing failure: what the Phase III progesterone studies can teach about TBI clinical trials. Brain Inj. 29, 1259-1272.

50. Schwamm, L.H. (2014). Progesterone for traumatic brain injury: resisting the siren's song. N. Engl. J. Med. 371, 2522-2523.

51. git.[Computer software]. (2020). https://git-scm.com/downloads (Last accessed October 23, 2020).

52. git-annex. [Computer software]. (2020). https://git-annex.branchable .com/ (Last accessed October 23, 2020).

53. csvkit. [Computer software]. (2020). https://github.com/wireservice/csvkit (Last accessed October 23, 2020).

54. Docker. [Computer software]. (2020). https://docs.docker.com/getdocker/ (Last accessed October 23, 2020)

55. OpenDistro for Elasticsearch. [Computer software]. (2020). https:// opendistro.github.io/for-elasticsearch/downloads.html (Last accessed October 23, 2020).

56. OpenDistro for Elasticsearch Kibana. [Computer software]. (2020). https://opendistro.github.io/for-elasticsearch/downloads.html (Last accessed October 23, 2020).

57. Logstash. [Computer software]. (2020). https://opendistro.github.io/forelasticsearch/downloads.html (Last accessed October 23, 2020).

58. Python. (2020). Python language interpreter [Computer Software] Version 3.8.6. https://www.python.org/ (Last accessed October 23, 2020).

59. RStudio Team (2020). RStudio: Integrated Development for R. RStudio, PBC, Boston, MA, http://www.rstudio.com/

60. Pennsylvania Trauma Systems Foundation Operational Manual for the Database Collection System. (2018). https://www.ptsf.org/wp-content/ uploads/2020/10/7-25-18_FINAL_2018_PTOS_Manual.pdf (Last accessed August 1, 2020)

61. Weihs, C., Ligges, U., Luebke, K., and Raabe, N. (2005). klaR analyzing German business cycles, in: Data Analysis and Decision Support. D. Baier, R. Decker, and L. Schmidt-Thieme (eds). Berlin: Springer-Verlag, pps. 335-343. 
62. Steinley, D. (2006). K-means clustering: a half-century synthesis. Br. J. Math Stat. Psychol. 59, 1-34.

63. Chang, J., and Sarkar, I.N. (2019). Using unsupervised clustering to identify pregnancy co-morbidities. AMIA Jt. Summits Transl. Sci. Proc. 2019, 305314.

64. Huang, Z. (1998). Extensions to k-means algorithm for clustering large data sets with categorical values. Data Min. Knowl. Discov. 2, 283-304.

65. Ramanathan, D.M., McWilliams, N., Schatz, P., and Hillary, F.G. (2012). Epidemiological shifts in elderly traumatic brain injury: 18-year trends in Pennsylvania. J. Neurotrauma 29, 1371-1378.

66. Germolec, D., Shipkowksi, K., Frawley, R., and Evans, E. (2018). Markers of inflammation, in: Immunotoxicity Testing: Methods and Protocols, 2nd ed. J. DeWitt, C. Rockwell, and C. Bowman (eds). New York, NY: Humana Press, pps. 57-79.

67. Ferrucci, L., and Fabbri, E. (2018). Imflammageing: chronic inflammation in ageing, cardiovascular disease, and frailty. Nat. Rev. Cardiol. 15, 505522.

68. Bektas, A., Schurman, S., Sen, R., and Ferrucci, L. (2018). Aging, inflammation, and the environment. Exp. Gerontol. 105, 10-18.

69. Bodnar, C.N., Morganti, J.M., and Bachstetter, A.D. (2018). Depression following a traumatic brain injury: uncovering cytokine dysregulation as a pathogenic mechanism. Neural Regen. Res. 13, 1693-1704.

70. Miller, A.H., Maletic, V., and Raison, C.L. (2008). Inflammation and its discontents : the role of cytokines in the pathophysiology of major depression. BPS 65, 732-741.

71. Sundman, M.H., Chen, N.K., Subbian, V., and Chou, Y.H. (2017). The bidirectional gut-brain-microbiota axis as a potential nexus between traumatic brain injury, inflammation, and disease. Brain Behav. Immun. 66 , 31-44.

72. Sendroy-Terrill, M., Whiteneck, G.G., and Brooks, C.A. (2010). Aging with traumatic brain injury: cross-sectional follow-up of people receiving inpatient rehabilitation over more than 3 decades. Arch. Phys. Med. Rehabil. 91, 489-497.

73. Jassam, Y., Izzy, S., Whalen, M., McGavern, D., and El Khoury, J. (2017). Neuroimmunology of traumatic brain injury: time for a paradigm shift. Neuron 95, 1246-1265.

74. Isokuortti, H., Iverson, G.L., Kataja, A., Brander, A., Öhman, J., and Luoto, T.M. (2016). Who gets head trauma or recruited in mild traumatic brain injury research? J. Neurotrauma 33, 232-241.

75. Luoto, T.M., Tenovuo, O., Kataja, A., Brander, A., Öhman, J., and Iverson G.L. (2013). Who gets recruited in mild traumatic brain injury research? J. Neurotrauma 30, 11-16.

76. Atsumi, H., Sorimachi, T., Honda, Y., Sunaga, A., and Matsumae, M. (2019). Effects of pre-existing comorbidities on outcomes in patients with chronic subdural hematoma. World Neurosurg. 122, e924-e932.

77. Bliemel, C., Buecking, B., Oberkircher, L., Knobe, M., Ruchholtz, S., and Eschbach, D. (2017). The impact of pre-existing conditions on functional outcome and mortality in geriatric hip fracture patients. Int. Orthop. 41, 1995-2000.

78. Garza, N., Toussi, A., Wilson, M., Shahlaie, K., and Martin, R. (2020). The increasing age of TBI patients at a single Level 1 trauma center and the discordance between GCS and CT Rotterdam scores in the elderly. Front. Neurol. 11, 1-7.

79. Thompson, H., McCormich, W., and Kagan, S. (2006). Traumatic brain injury in older adults: epidemiology, outcomes, and future implications. J. Am. Geriatr. Soc. 54, 1590-1595.

80. Stein, D.M., Kozar, R.A., Livingston, D.H., Luchette, F., Adams, S.D., Agrawal, V., Arbabi, S., Ballou, J., Barraco, R.D., Bernard, A.C., Biffl, W.L., Bosarge, P.L., Brasel, K.J., Cooper, Z., Efron, P.A., Fakhry, S.M., Hartline, C.A., Hwang, F., Joseph, B.A., Kurek, S.J., Moore, F.A., Mosenthal, A.C., Pathak, A.S., Truitt, M.S., and Yelon, J.A. (2018). Geriatric traumatic brain injury: what we know and what we don't. J. Trauma Acute Care Surg. 85, 788-798.

81. Peters, M.E., and Gardner, R.C. (2018). Traumatic brain injury in older adults: do we need a different approach? Concussion 3, CNC56.

82. Trivedi, R.B., and Humphreys, K. (2015). Participant exclusion criteria in treatment research on neurological disorders: are unrepresentative study samples problematic? Contemp. Clin. Trials 44, 20-25.

83. Walker, W.C., Stromberg, K.A., Marwitz, J.H., Sima, A.P., Agyemang, A.A Graham, K.M., Harrison-Felix, C., Hoffman, J.M., Brown, A.W., Kreutzer, J.S., and Merchant, R. (2018). Predicting long-term global outcome after traumatic brain injury: development of a practical prognostic tool using the Traumatic Brain Injury Model Systems national database. J. Neurotrauma 35, 1587-1595.

84. Dijkers, M.P., Marwitz, J.H., and Harrison-Felix, C. (2018). Thirty years of National Institute on Disability, Independent Living and Rehabilitation Research Traumatic Brain Injury Model System Centers research: an update. J. Head Trauma Rehabil. 33, 363-374.

85. Brenner, E.K., Grossner, E.C., Johnson, B.N., Bernier, R.A., Soto, J., and Hillary, F.G. (2020). Race and ethnicity considerations in traumatic brain injury research: incidence, reporting, and outcome. Brain Inj. 34, 799-808

86. Alzheimer's Association. (2020). 2020 Alzheimer's disease facts and figures. Alzheimer's Dement. DOI: 10.1002/alz.12068.

Cite this article as: Dell, KC, Grossner, EC, Staph, J, Schatz, P, and Hillary, FG (2021) A population-based study of pre-existing health conditions in traumatic brain injury. Neurotrauma Reports 2:1, 255269, DOI: $10.1089 /$ neur.2020.0065.

$\begin{aligned} & \text { Abbreviations Used } \\ & \mathrm{ADHD}=\text { attention deficit hyperactivity disorder } \\ & \mathrm{ANOVA}=\text { analysis of variance } \\ & \mathrm{CDC}=\text { Centers for Disease Control and Prevention } \\ & \mathrm{COPD}=\text { chronic obstructive pulmonary disease } \\ & \mathrm{CVD}=\text { cardiovascular disease } \\ & \mathrm{ED}=\text { emergency department } \\ & \mathrm{FIM}=\text { functional independence } \\ & \mathrm{GCS}=\text { Glasgow Coma Scale } \\ & \mathrm{ICD} 9=\text { International Classification of Diseases, 9th Revision } \\ & \mathrm{ICU}=\text { intensive care unit } \\ & \mathrm{LTC}=\text { long term care facility } \\ & \mathrm{mSTBI}=\text { moderate-to-severe TBI } \\ & \mathrm{mTBI}=\text { mild TBI } \\ & \mathrm{NCCDPH}=\text { National Center for Chronic Disease Prevention } \\ & \mathrm{NTDB}=\text { National Trauma Data Bank } \\ & \mathrm{OR}=\text { odds ratio } \\ & \mathrm{PEC}=\text { pre-existing health conditions } \\ & \mathrm{PTOS}=\text { Pennsylvania Trauma Outcomes Study } \\ & \mathrm{PTSF}=\text { Pennsylvania Trauma Systems Foundation } \\ & \mathrm{SD}=\text { standard deviation } \\ & \mathrm{TBI}=\text { traumatic brain injury } \\ & \mathrm{WSS}=\text { within-cluster sum of squares } \\ &\end{aligned}$

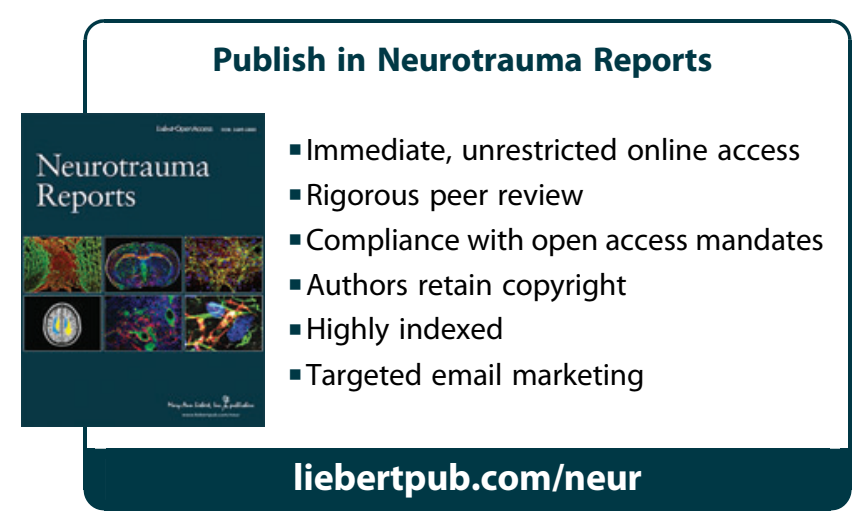

